\title{
Scutellaria Barbata D.Don (SBD) Extracts Suppressed Tumor Growth, Metastasis and Angiogenesis in Prostate Cancer Via PI3K/Akt Pathway
}

\section{Dongya Sheng}

Yueyang Hospital of Integrated Traditional Chinese and Western Medicine, Shanghai University of Traditional Chinese Medicine

\section{Bei Zhao}

Institute of Interdisciplinary Integrative Medicine Research, Shanghai University of Traditional Chinese Medicine, Shanghai, China

\section{Wenjing Zhu}

Yueyang Hospital of Integrated Traditional Chinese and Western Medicine, Shanghai University of

Traditional Chinese Medicine

\section{Tiantian Wang}

Yueyang Hospital of Integrated Traditional Chinese and Western Medicine, Shanghai University of

Traditional Chinese Medicine

\section{yu peng ( $\nabla$ drypeng@sina.com)}

Yueyang Hospital of Integrated Traditional Chinese and Western Medicine, Shanghai University of Traditional Chinese Medicine

\section{Research Article}

Keywords: Prostate cancer, Scutellaria barbata D.Don, PI3K/AKT, Metastasis, Apoptosis, Angiogenesis

Posted Date: December 20th, 2021

DOl: https://doi.org/10.21203/rs.3.rs-1105578/v1

License: (c) (1) This work is licensed under a Creative Commons Attribution 4.0 International License. Read Full License 


\section{Abstract}

Background: Scutellaria barbata D.Don (SBD) is derived from the dried whole plant of Labiate that has been widely used to treat patients with multiple cancer. It was previously reported that the ethanol extract of SBD is able to promote apoptosis, and inhibit cell proliferation and angiogenesis in cancer.

Materials and methods: CCK8, Edu assays and colony formation assay were performed to assess the effect of SBD on PCa cell growth. Effect of SBD on apoptosis and cell cycle was detected by flow cytometry. Transwell and wounding healing assay were performed to detected the invasion and migration activities of PCa cells. Western blot was employed to detect the protein expression. 2RRV1 mouse xenograft model was established to detect the effect of SBD on prostate cancer. Angiogenesis was analysed by coculturing PCa cell lines and HUVECs.

Results: The results showed that SBD induced a significant decrease in cell viability and clonogenic growth in a dose-dependent manner. SBD induced cell apoptosis and cell cycle G2/M phase arrest by inactivating PI3K/AKT signalling pathway. Treatment with SBE also significantly decreased the cell migration and invasion via phenotypic inversion of EMT that was characterized by the increased expression of E-cadherin and Vimentin, and decreased expression of $\mathrm{N}$-cadherin, which could be partially attributed to inhibiting PI3K/AKT signalling pathway. Subsequently, using AKT inhibitor MK2206, we performed that PI3K/AKT are also involved in cell apoptosis and metastasis of PCa cells stimulated by SBE. In addition, to its direct effects on PCa cells, SBD also exhibited anti-angiogenic properties. SBD alone or conditioned media from SBD-treated PCa cells inhibited HUVEC tube formation on Matrigel without affecting HUVEC viability. Furthermore, 22RV1 xenograft C57BL/ 6 mice treated with SBE in vivo showed a significant decrease in tumour size and tumour weight without toxicity. In addition, administration with medium- or high-dose of SBE significantly inhibited the cell proliferation and promoted the damage of tumour tissues.

Conclusions: Collectively, our in vitro and in vivo findings suggest that SBE had the potential to develop into a safe and potent alternative therapy for PCa patients.

\section{Introduction}

Prostate cancer $(\mathrm{PCa})$ is a common male genitourinary tumours and poses threat to the health of men worldwide[1, 2]. It is a crucial health problem globally, which brings a huge economic burden to society[3]. Despite conventional therapeutic approaches aim to prolong progression-free survival, patients diagnosed with PCa are still at risks of recurrences, low postoperative survival rates, high metastasis rate and harmful side-effects and led to resistance[4].

The epithelial-to-mesenchymal transition (EMT) is considered a hallmark of the aggressive invasion and metastasis of advanced tumours[2]. In previous studies, EMT can bring about the malignant transformation of $\mathrm{PCa}$, and thus leading to its invasion and migration[5]. 
Currently, traditional Chinese medicine (TCM) formulas are widely applied to treat advanced malignant tumours, because of its good efficacy and few side effects[6]. Several clinical trials have shown that TCM treatment significantly improves the nutritional status and quality of life of patient with PCa.

Tumour angiogenesis, as a significant cancer hallmark, play a crucial role in tumour progression, including rapid growth, recrudesce and metastasis[7, 8]. Through excessive secretion of pro-angiogenic factor, tumour cell continuously activate endothelial cells to 'sprout ' in the original blood vessels to form new vascular structures[9]. At the same time, angiogenesis supplies the fundamental nutrients and oxygen to cancer cells, and also a way for metastasis[10]. Therefore, inhibiting tumour angiogenesis has been an effective strategy for cancer therapy since decades ago.

The application of traditional Chinese medicine (TCM) in cancer treatment has been recorded in Chinese medical texts and pubications for more than two millennium. Currently, traditional Chinese medicine has become more common due to its safety and clinical efficacy for $\mathrm{PCa}$, in particular its synergistic effects and decrese side effects and toxicity. SBD, one of the well-known Chinese herbs medicines, has been widely used clinically for treating malignant cancer for many years. As mentioned earlier, SBE induced cancer cell apoptosis by activating MAPK and ER stress signaling pathway, restrained tumor angiogenesis via inhibiting Hedgehog signaling pathway, and dinimished G2 arrest in lung cancer cells by regulating mutiple signaling pathways related with the cell cycle checkpoint enzymes $[5,11-13]$. These studies have been indicated that SBD is a potential drug in anti-tumor.. Nevertheless, the underlying molecular mechanism of SBD in PCa and cancer metastasis have not yet been elucidated.

In present study, we investigated the anti-tumor, anti-metastasis activities of SBD and its mechanism by using a series of in vitro assays and in vivo mouse model. Subsequently, we investigated the antiangiogenic effect of SBD on human umbilical vein endothelial cell (HUVEC). This study will offer a new clue on the clinical application of SBD.

\section{Materials And Methods}

\section{Preparation of SBD}

The raw herb was purchased by Yueyang Hospital of integrated Traditional Chinese and Western Medicine(Lot: 210526;Place of production: Shanxi). And they were identified by Liuhong, from the department of pharmacology, Yueyang Hospital of integrated Traditional Chinese and Western Medicine. Preparation of SBD was established as previously described. Briefly, the dried rhizomes of SBD were ground into powder, which was then extracted with double-distilled water by reflux extraction for 1.5 h/time 2times. Ninety-five percent ethanol was added to the mixed extract to lower the final concentration of ethanol to $85 \%(\mathrm{v} / \mathrm{v})$. The precipitated polysaccharide component was taken out by the filtration device. A rotary evaporator concentrated the resulting solution at $50^{\circ} \mathrm{C}$ under condition of lower pressure. Eventually, the extract was dissolved again in methanol for carrying out liquid chromatography (HPLC) analysis[14]. 


\section{Analysis of SBD}

SBD was analysed on uadrupole time-of-flight mass spectrometry (UPLC-Q- TOF-MS). The system was completed on a Waters ACQUITY UPLC HSS C18 column and the column temperature was $25^{\circ} \mathrm{C}$. Mass spectrometry analysis was conducted on an AB Sciex Triple TOF® 4600 system equipped with an ESI source. The detection wavelength was measured at $254 \mathrm{~nm}$.

\section{Cell culture and reagents}

Human umbilical vein endothelia cells (HUVECs), human PCa cell lines(PC-3 and DU145)and Mouse PCa cell line (22RV1) were cultured in DMEM supplemented 10\% fatal bovine serum (FBS) and 1\% penicillinstreptomycin at $37^{\circ} \mathrm{C}$ and $5 \% \mathrm{CO}_{2}$. Cells were treated with $\mathrm{SBD}$ in fresh medium at different concentration, and the control group employed an equal volume of DMSO.

\section{Cell viability}

Cells were exposed to stepwise increasing concentrations of SBD. After treatment with different concentration of SBD, the cell viability assay was detected using a CCK-8 kit (APE-BIO, MA, USA) follow the manufacture' s instruction. Briefly, after $24 \mathrm{~h}$ or $48 \mathrm{~h}, \mathrm{OD}$ value was measured by using CCK8 regent. The cell viability rate calculation formula is : Cell viability $(\%)=O D$ value of the experimental/OD value of the control group $\times 100 \%$.

\section{Cell apoptosis assay}

After 48h of SBD treatment, PCa cells were harvested. Cell apoptosis was determined by an Annexin VFITC/PI Apoptosis Detection Kit (Biolegend, MA, USA). All results were analyzed by flow cytometer (Beckman Coulter, MA, USA).

\section{Cell clonogenicity assay}

Cells were divided into three group: a control group, a $50 \mu \mathrm{g} / \mathrm{mL}$ SBD and a $100 \mu \mathrm{g} / \mathrm{mL} \mathrm{SBD}$ and maintained at the SBD containing completed medium $10 \mathrm{~d}$. Subsequently, the cells were fixed by $4 \%$ methanol for $20 \mathrm{~min}$, then stained with crystal violet for $20 \mathrm{~min}$. Glacial acetic acid was added into plate, subsequently the OD value of each well at $590 \mathrm{~nm}$ was measured by microplate reader.

\section{Cell cycle analysis}

Cells were stimulated with SBD (50 and $100 \mu \mathrm{g} / \mathrm{mL}$ ) for $48 \mathrm{~h}$, and then fixed by $70 \%$ ethanol at $-20^{\circ} \mathrm{C}$ overnight. Afterwards, cells were washed with phosphate-buffered saline (PBS), and incubated with 500 $\mathrm{uL}$ of propidium iodide (PI) (BD Phamingen, USA) staining solution for $15 \mathrm{~min}$. Afterwards, cells were detected under a FACS-calibur flow cytometry (Beckman-Coulter, Shanghai, China).

\section{Cell proliferation analysis}

Cell proliferation was measured using EDU solution (Beyotime, Shanghai, China) for $2 \mathrm{~h}$, and then stained with $1 \times$ Hoechst 33342 solution (Beyotime, Shanghai, China) for 10 min at $25^{\circ} \mathrm{C}$. Morphological changes 
were observed using a fluorescence microscope. (Magnification, $\times 100$ ).

\section{Wound healing assay}

The cells in each well were linearly scraped by a $10 \mu \mathrm{L}$ of sterile pipette tip. Afterwards, each well was added fresh medium with SBD, and incubated for $48 \mathrm{~h}$. The images of cross were photographed under the microscope.

\section{Tube formation assay}

PCa cells were cultured, and treated without or with SBD or phorbol 12-myristate 13-acetate (PMA) at the different concentration for $48 \mathrm{~h}$. The conditioned media (CM) was the collected and applied to HUVEC. Matrigel matrix gel ( $50 \mu \mathrm{L} /$ well) was added into a 96 -well plate to incubate for $30 \mathrm{~min}$. Subsequently, $1 \times 10^{4}$ HUVECs were dispersed in the $\mathrm{CM}$ and then seeded on the Matrigel layer for incubation at $37^{\circ} \mathrm{C}$ in $5 \% \mathrm{CO}_{2}$ for $4 \mathrm{~h}$. Then photography was performed with a microscope.

\section{Cell migration and invasion assays}

For the cell invasion assay, chambers were pre-coated with Matrigel (BD Biosciences, San Jose, CA). Cells stably transfected with different constructs were starved overnight, and then seeded in the upper chamber (corning, Billerica, MA) at a density of $1 \times 10^{5}$ cells $/ \mathrm{mL}$ in $200 \mu \mathrm{L}$ of medium without FBS. Meanwhile, lower chamber plated into $600 \mu \mathrm{L}$ of medium with $15 \mathrm{~mL}$ FBS. 24 hours later, when the incubation was finished, A cotton swab was used to clear non- migrating (or non- invading) cells in the upper chamber. We quantified invading cells by using manual counting.

\section{Western blotting assay}

Cells and tumour tissue were harvested and lysed with RIPA buffer. Proteins were transferred to polyvinylidene fluoride (PVDF) membranes for $90 \mathrm{~min}$. In this study, primary antibodies used as follow: Cleaved-caspase-3, Bax, Bcl-2, p-AKT, AKT, p-PI3K, PI3K, E-cadherin, N-cadherin and Vimentin. After washing, the secondary antibodies were used to visualize the blot under the Bio-Rad imaging system.

\section{Tumor xenograft in mice model}

Male C57BL/6 mice from the Shanghai University of traditional Chinese Medicine Laboratory Animal Management Department. All mice were conducted according to the Guide for the Care and Use of Laboratory Animals of Shanghai University of traditional Chinese Medicine Laboratory Animal Management Department, as approved by the Animal care Committee of Shanghai Province, China(Approval No. P2SHUTCM210715001). The mice were subcutaneously engrafted in the right-hind flank with $1 \times 10^{6} 22 \mathrm{RV} 1$ cells (day 0 ). Mice were randomly allocated to 4 groups $(n=5)$, including the control group ( $0.9 \%$ saline), SBD medium-dose (50 mg/kg), SBD high-dose group ( $100 \mathrm{mg} / \mathrm{kg})$. SBD was given orally once a day. The body weight and tumour size of the mice were monitored every 2 days. Tumour volumes $(V)$ were calculated using the formula $V=\left(L \times D^{2}\right) / 2$, where $L$ is the greatest diameter and $d$ is the smallest diameter of the tumour. During the experiment, the mice did not show any 
discomfort. After $16 \mathrm{~d}$, the tumours were extracted from sacrificed mice to weighed. All sections of this research adhere to the ARRIVE Guidelines for reporting animal research. A completed ARRIVE Guidelines checklist is included in Additional file 1.

\section{Histological analysis}

Tumour tissue were perfused with $4 \%$ paraformaldehyde before embedded in paraffin and sectioned at a thickness of $3 \mu \mathrm{m}$ thick. For H\&E staining, the tissue was sliced after deparaffinization, stained and fixed on a glass slide with neutral gum. Tumour sections were immunohistochemically stained with Ki67, Ecad, N-cad, and Vim antibodies according to the steps of manufacturer. After the above steps were completed, took photos using the microscope.

\section{Statistical analysis}

All data are presented as the Mean \pm Standard Deviation of more than 3 independent experiments. Unpaired Student's t-test was used for two experimental groups and one-way ANOVA test were used for multiple groups. A two-tailed value of $p<0.05$ was considered statistically significant. Statistics were done using GraphPad Prism 8.0.

\section{Results}

\section{The chemical components of SBD}

The related chromatograms were shown in Fig. 1.

\section{SBD inhibited cell proliferation and induced cell cycle arrest in PCa cells}

The cytotoxicity of SBD on PC-3 and DU145 cell lines was detected by CCK8 assay. Cells were exposed to various concentration of SBD for $24 \mathrm{~h}$ (Figure. 2a) and $48 \mathrm{~h}$ (Figure. 2b). According to the results of cell viability, SBD showed an obvious cytotoxic effect against PC-3 and DU145 cell lines in a time and concentration dependent manner, with $\mathrm{IC}_{50}$ of $98.44 \mu \mathrm{g} / \mathrm{mL}$ and $118.2 \mu \mathrm{g} / \mathrm{mL}$, in $24 \mathrm{~h}$ respectively, and $56.39 \mu \mathrm{g} / \mathrm{mL}$ and $66.51 \mu \mathrm{g} / \mathrm{mL}$ in $48 \mathrm{~h}$, respectively. SBD had marginally anti-tumour effects on PC-3 than on DU145.

Afterwards, the cytotoxic phenotypes in PC-3 (Figure. 2c) and DU145 cell lines (Figure. 2d) were detected under EdU incorporation staining. The observations demonstrated that SBD concentration-dependently decreased the radio of EdU-positive nuclei in both PCa cell lines.

Immortal proliferation is a crucial feature of tumour and timing of proliferation rely on the transition speed of cell cycle[15]. It has been observed that cell cycle plays an important role in the progression of PCa cells[16]. The effect of SBD on cell cycle of PC-3 (Figure. 2e) and DU145 cell lines (Figure. 2f) was detected. The data demonstrated that most of the cells were arrested in the $S$ and $G 2$ phases after treatment with SBD in PC-3 cells. Except for an obvious increase in S and G2 phases cell population, SBD treatment decreased the $\mathrm{G} 1$ phase cell population $(p<0.05)$. Consistent with PC3 cells, treatment with SBD resulted in an apparent increase in the G2 phase cell population in the DU145 cell $(p<0.05)$. In 
addition, the colony formation experiment indicated that SBD suppressed the colony formation of PCa cells (Figure. $2 \mathrm{~g} \& 2 \mathrm{~h}$ ).

Taken together, the inhibitory effect of SBD on the cell proliferation and colony formation might partially due to the cell cycle arrest at $\mathrm{G} 2 / \mathrm{M}$ phase.

\section{The PI3K/AKT signaling pathway participates in SBD-induced PCa cell apoptosis}

Increasing apoptosis is the main index to assess the efficacy of antitumor drugs[17]. We then investigate whether SBD has the ability to induce apoptosis the biological function of SBD in apoptosis, the cell apoptosis in SBD - treated PC-3 (Figure. 3a) and DU145 cells (Figure. 3b) was analyzed with flow cytometry. The percentage of apoptosis cells was dramatically increased in PC3 and DU145 cells treated with SBD when compared with their controls $(p<0.05)$.

Thus, from prior reports, we hypothesized that SBD could constrain the expression of a panel of proteins associated with cellular apoptosis. The Western blot results that SBD treated PCa cells boosted expression of activated Caspase-3, proving that apoptosis was the major mechanism of SBD -induced growth inhibition in PCa cells. After that, we examined that impact of SBD on the Bcl-2 family of proteins involved in apoptosis. There was an increase in the expression of pro-apoptotic Bax protein in both PC3 and DU145 cells treated with SBD. In addition, treatment with SBD for $48 \mathrm{~h}$ down-regulated the expression of anti-apoptotic Bcl-2 protein in PCa cells (Figure. 3c).

The abnormal activation of PI3K/AKT signalling pathway is very frequently observed in PCa. Furthermore, SBD significantly inhibited the phosphorylation of AKT and PI3K in PCa cells (Figure. 3d), which are consistent with the roles for PI3K/AKT signalling pathway in the regulation of cell apoptosis. These dates demonstrated that SBD promote PCa cells apoptosis via inactivation of PI3K/AKT signalling pathway.

\section{SBD inhibits migration, invasion, EMT progression and TME-angiogenesis}

The metastasis and invasion are closely related to the malignant degree and prognosis of the tumour[18]. According to the wound-healing assay, SBD significantly inhibited tumour cell migration in both PC3 (Figure. 4a) and DU145 cells (Figure. 4b) in a dose-dependent manner. To determine if SBD could supress migration and invasion of PCa cells, transwell assays were employed in PC3 and DU145 cells. As expected, treatment with SBD for $48 \mathrm{~h}$ reduced the number of invaded cells remarkably $(p<0.05$, Figure. $4 c \& 4 d)$. These results indicated that SBD decelerate PCa cells migration and invasion.

As reported in previous studies, EMT is inseparably associated with metastasis of tumour. To further study the potential mechanism of SBD on its anti-metastatic effect, we next assessed the expression of EMT-related protein though western blot. As indicated in Figure. 4e, the expression of the epithelial marker $\mathrm{E}$-cadherin and Vimentin was significantly up-regulated by SBD, while the expression of $\mathrm{N}$-cadherin was down-regulated remarkably in the SBD -treated cells compared to the control cells $(p<0.05$, Figure. $4 e)$. 
Collectively, these results showed that SBD can affect cancer cell invasion, migration and EMT-related protein.

\section{Blockade of AKT resulted in inhibition of SBD-mediated cell proliferation and migration}

To further determine the involvement of the PI3K/AKT pathway in the inhibition of tumour cell growth by SBD, PC-3 and DU145 cells were pre-treated with $10 \mu \mathrm{M}$ MK2206 (AKT-specific inhibitor) for $6 \mathrm{~h}$, followed by $48 \mathrm{~h}$ of SBD treatment. After $48 \mathrm{~h}$, cell viability was analysed using CCK-8 assay. Of note, co-treatment with SBD had no significant effect on cell viability compared with those treated alone with SBD (50 $\mu \mathrm{g} / \mathrm{mL}$ ) in PC-3 (Figure. 5a) and DU145 cells (Figure. 5b). In addition, co-treatment with SBD did not further decrease the expression of p-AKT $(p>0.05)$.

To further investigate if the cell invasion inhibitory effect of SBD is dependent on AKT/PI3K signalling pathway, MK2206 was pre-treated on PCa cells. The results showed that PC-3 (Figure. 5c) and DU145 cells (Figure. $5 \mathrm{~d}$ ) migration had no significant difference when compared with SBD $(50 \mu \mathrm{g} / \mathrm{mL})$ treatment alone $(p>0.05)$. These results above indicated that SBD inhibited cell viability and invasion in a mechanism dependent on the AKT/PI3K pathway partially.

\section{SBD inhibited EMT via AKT/PI3K pathway signaling}

Based on the abovementioned results, SBD may regulate EMT through PI3K/AKT signaling pathway. According to the results of western blot, we found MK2206 reversed effects of SBD on expression of $p$ AKT, N-cadherin, E-cadherin and Vimentin on PC-3 and DU145 cells $(p<0.05)$ (Figure. 6a \& b). Collectively, these results suggested that treatment with SBD may partially inactivate PI3K/AKT signalling pathway, thereby inhibiting the cell proliferation and invasion, as well as preventing PCa cells from EMT progression.

\section{SBD suppressed tumor growth in 22RV1 cell xenograft mice model}

Next, to investigate the anti-tumour effect of SBD on the inhibition of PCa in vivo, we established 2RRV1 subcutaneous xenografts. We explored the effects of Low-dose (SBD-L), Medium-dose (SBD -M), and High-dose (SBD -H) of SBD treatment on tumour growth. As shown in Figure. 7a \& 7b \& 7c, administration of SBD in 22RV1-bearing mice markedly inhibited tumour volume and the tumour weight. Our results showed that there are significant different between the SBD treatment and the control group. H\&E and proliferating cell-related antigen Ki67 staining were conducted to explore the growth level of the xenografts. Ki67-positive rate was decreased markedly in the SBD-treated group with medium and high dose (Figure. 7d \& 7e), confirmed that subcutaneous tumour proliferation was significantly reduced in the SBD-M and SBD-L treatment group.

\section{SBD inhibits AKT/PI3K and EMT progression in vivo}

Based on the results of the in vitro study, western blotting and immunohistochemical (IHC)assays were used to detect the expression levels of EMT-related proteins in tumour tissues. As shown in Figure. 8a, 
administration with SBD-M and SBD-H reduced the phosphorylation levels of PI3K and AKT remarkably ( $p$ $<0.05$ ) in tumour tissues. In addition, according to the IHC staining results, the expression of E-cadherin and Vimentin was significantly up-regulated, while the expression of $\mathrm{N}$-cadherin was down-regulated remarkably in SBD-M and SBD -H groups when compared to the control groups $(p<0.05$, Figure. 8b)

\section{SBD blocks tube formation of human umbilical vein endothelial cells}

Angiogenesis is a main process in cancer development and plays a vital role in cancer growth and metastasis. The effects of SBD on angiogenesis in vitro was determined by tube formation and migration experiments. We collected the SBD in the supernatant of tumour cells and subject it to HUVECs. There are significance difference between complete media-treated HUVECs and CM from PCa cells-treated HUVECs, whilst CM from SBD-treated PCa cells obviously diminished the ability of tube formation of HUVEC in concentration-dependent manner (Figure 9A). We next investigated whether SBD-mediated inhibition of the tube formation in HUVEC is due to the drug's cytotoxicity. The viability and survival rate of HUVEC without or with SBD treatment for $48 \mathrm{~h}$ did not change significantly, which ruled out the possibility of drug cytotoxicity $(p>0.05)$ (Figure. 9B). It is well known that the PKC activator PMA is able to induce the tube formation of HUVECs cells[19]. When HUVEC were treated with $10 \mathrm{nM} \mathrm{PMA}$, they formed the typical cobblestone morphology characteristic, which was greatly weakened by SBD treatment (Figure. 9C). These results indicated that SBD inhibited angiogenesis by reducing tube formation of vascular endothelial cells rather than apoptosis.

\section{Discussion}

As a global health threat, $\mathrm{PCa}$ ranks second in cancer-associated mortality in men in western countries[20]. Currently, the standard treatment for PCa is mainly treated by surgical resection, radiotherapy, and chemotherapy, the long-term effect is still not good, and usually lead to serious sideeffects. Nowadays, alternative therapies using Chinese herbal extracts and formulas propose new methods to the administration of anti-PCa [21-23]. Thus, active ingredients in extracts derived from plants have synergistic interactions, which may play a role in fighting against cancer. In recent years, Chinese medicine possess excellent bioactivity with low side-effect in the clinical application of many diseases. Despite the accomplishments of researches into finding anti-cancer therapy with Chinese medicines, these medicines are far from being widely applied in the treatment of PCa and there is still a long way to go.

In the current study, we demonstrated that SBD inhibited the growth of PC3 and DU145 in a dosedependent manner. Clonogenic assay further validated the inhibitory effect of SBD on PCa cells. Meanwhile, we recapitulated the anti-tumour efficacy of SBD in vivo. There were no adverse effects in mice during the experiment. These findings implied that SBD had the potential to develop into a safe and effective alternative therapy for PCa patients.

It is well known that he Bcl-2 family proteins which are one of the main factors participate in the apoptosis pathway since they modulate apoptosis by regulating mitochondrial membrane 
permeability[24]. Bcl-2 is a anti-apoptotic protein, while Bax is an apoptosis inducer[25]. Caspase-3 is the key factor protein as a downstream effector of apoptosis and the increased Caspase-3 level is always used as an indicator of apoptosis[26]. In order to evaluate the molecular mechanism of SBD against PCa, we performed western blot analysis of Cleaved-Caspase-3, Bcl-2 and Bax in PCa cells. Herein, treatment with SBD increased the activities of Cleaved-Caspase-3, enhanced the expression levels of Bax protein, and down-regulated the expression level of Bcl-2 protein, characterizing the extrinsic pathway of cell apoptosis. In addition, the anti-apoptotic function of AKT has been related to inactivation of Bax and Cleaved-caspase-3, as well as overexpression of Bcl-2[27].

Many evidences indicate that PI3K/Akt signalling pathway is an important regulator involved in cell proliferation and metastasis[28, 29]. Interestingly, we observed the inactivation of PI3K/AKT signalling pathway in PCa cell treated by SBD. The pro-apoptotic and G2/M phase arrest activities of SBD in PCa cells could partially been caused by indicating that suppression of PI3K/AKT signalling pathway.

EMT is a complex process involving endothelial cell proliferation, differentiation, migration. Increased of migration and invasion are actively related to the EMT progression, which is characterized by inhibiting the expression of epithelial markers (E-cadherin and Vimentin) and inducing the expression of mesenchymal markers (N-cadherin) [30-32]. After going through the EMT progress, not only the connection between cells, the contract of cell to matric, but also the normal epithelial polarity disappears among PCa cells. Meanwhile, they gain mesenchymal features to migrate and invade nearby matrix. [33, 34]. This process is driven by activating and/or crosstalk between several signalling pathways. Several studies have shown that EMT-related proteins in tumour tissues, especially the EMT marker, indicate a poor survival of PCa patients.

Upon activation of PI3K/AKT signalling pathway is disposed to induce the expression levels of EMTrelated marker[35]. In addition, the PI3K/AKT signalling pathway is a vital player in EMT in cancer cells[36]. Consequently, our further goal for this investigation was to elucidate potential molecular mechanism of actions for the SBD treatment. Notably, PI3K inhibitor plays a vital role in PCa cell apoptosis and migration by inhibiting the PI3K/AKT signalling pathway[37]. PCa cells were treated with SBD and an PI3K inhibitor (MK2206), followed by incubation for $48 \mathrm{~h}$ and the change in cell viability, invasion and EMT were all evaluated. Our results showed that compared with SBD treatment alone, SBD and MK2206 co-treatment have no significant on apoptosis, invasion and the expression of EMT-related protein in PCa cells, which suggested that SBD could inhibit the EMT in PCa cells through attenuating $\mathrm{PI} 3 \mathrm{~K} / \mathrm{AKT}$ signalling pathway.

Antiangiogenic therapy is an critical strategy for the treatment of PCa. Interaction between PCa cells and HUVEC played promoting effects on cell migration between each other, which also contributed to mosaic vessels formation[38]. However, these effects were supressed by SBD treatment. In addition, PMA promoted tube formation of HUVEC, while these promoted effects were also remarkably inhibited by SBD treatment. 


\section{Conclusion}

Taken together, our study revealed that SBD could exert the anti-cancer activity in PCa cells, both in vitro and in vivo. SBD inhibited PCa cells growth, migration and invasion via inducing cell apoptosis, G2/M phase cell cycle arrest, inhibiting EMT progression, which is characterized by attenuating PI3K/AKT signalling pathway. We also observed the anti-angiogenic effect of SBD. Accordingly, SBD could play the role as a potential adjuvant agent in treating PCa patients.

\section{Abbreviations}

SBD, Scutellaria barbata D.Don; PCa, Prostate cancer; TCM,Traditional Chinese Medicine ;p-AKT, phosphorylated AKT; PI3K, phosphoinositide 3-kinase; EMT, The epithelial-to-mesenchymal transition. CCK8هCell counting Kit-8.

\section{Declarations}

\section{Acknowledgments}

Not Applicable.

\section{Ethics approval}

Animal experiments were conducted according to the Guide for the Care and Use of Shanghai University of traditional Chinese Medicine Laboratory Animal Management Department, as approved by the Animal care Committee of Shanghai Province, China (Approval No. P2SHUTCM210715001).

\section{Authors' contributions}

DS conceived the study. DS and BZ planned experiments. DS, BZ and WZ helped with the animal experiments.DS, BZ and TW cultured the cells.DS and YP performed data analysis and constructed the figures. DS, BZ, WZ, TW, and YP wrote the manuscript, and all authors read and approved the manuscript.

\section{Competing interests}

The authors declare that they have no competing interests.

\section{Funding}

This study was supported by National Natural Science Foundation of China (No.81904070 and 81973729).

\section{Availability of data and materials}


The datasets used and analysed during the current study are available from the corresponding author on reasonable request.

\section{Author details}

${ }^{1}$ Yueyang Hospital of Integrated Traditional Chinese and Western Medicine, Shanghai University of Traditional Chinese Medicine. ${ }^{2}$ Institute of Interdisciplinary Integrative Medicine Research, Shanghai University of Traditional Chinese Medicine, Shanghai, China

\section{References}

1. Guo H, Luo H, Yuan H, Xia Y, Shu P, Huang X, Lu Y, Liu X, Keller ET, Sun D et al: Litchi seed extracts diminish prostate cancer progression via induction of apoptosis and attenuation of EMT through Akt/GSK-3ß signaling. Sci Rep 2017, 7:41656-41656.

2. Taki M, Abiko K, Ukita M, Murakami R, Yamanoi K, Yamaguchi K, Hamanishi J, Baba T, Matsumura N, Mandai M: Tumor Immune Microenvironment during Epithelial-Mesenchymal Transition. Clinical cancer research: an official journal of the American Association for Cancer Research 2021.

3. Adamaki M, Zoumpourlis V: Prostate Cancer Biomarkers: From diagnosis to prognosis and precisionguided therapeutics. Pharmacology \& therapeutics 2021, 228:107932.

4. Cornford P, van den Bergh R, Briers E, Van den Broeck T, Cumberbatch M, De Santis M, Fanti S, Fossati N, Gandaglia G, Gillessen S et al: EAU-EANM-ESTRO-ESUR-SIOG Guidelines on Prostate Cancer. Part II-2020 Update: Treatment of Relapsing and Metastatic Prostate Cancer. European urology 2021, 79(2):263-282.

5. Chen W, Gong K, Yang L, Dai J, Zhang Q, Wang F, Li X, Xi S, Du JJJoe: Scutellariabarbata D. Don extraction selectively targets stemness-prone NSCLC cells by attenuating SOX2/SMO/GLI1 network loop. 2021, 265:113295.

6. Chan HHL, Ng T: Traditional Chinese Medicine (TCM) and Allergic Diseases. Curr Allergy Asthma Rep 2020, 20(11):67.

7. Eelen G, Treps L, Li X, Carmeliet PJCr: Basic and Therapeutic Aspects of Angiogenesis Updated. 2020, 127(2):310-329.

8. Potente M, Gerhardt H, Carmeliet PJC: Basic and therapeutic aspects of angiogenesis. 2011, 146(6):873-887.

9. Fernández-Cortés M, Delgado-Bellido D, Oliver FJFio: Vasculogenic Mimicry: Become an Endothelial Cell "But Not So Much". 2019, 9:803.

10. Schnegg C, Yang M, Ghosh S, Hsu MJCr: Induction of Vasculogenic Mimicry Overrides VEGF-A Silencing and Enriches Stem-like Cancer Cells in Melanoma. 2015, 75(8):1682-1690.

11. Li W, Xiao HJD, metabolic syndrome, targets o, therapy: Scutellaria barbata D. Don Polysaccharides Inhibit High Glucose-Induced Proliferation and Angiogenesis of Retinal Vascular Endothelial Cells. $2021,14: 2431-2440$. 
12. Hnit S, Yao M, Xie C, Ge G, Bi L, Jin S, Jiao L, Xu L, Long L, Nie H et al: Transcriptional regulation of $\mathrm{G} / \mathrm{M}$ regulatory proteins and perturbation of $\mathrm{G} / \mathrm{M}$ Cell cycle transition by a traditional Chinese medicine recipe. 2020, 251:112526.

13. Zheng X, Kang W, Liu H, Guo SJljomm: Inhibition effects of total flavonoids from Sculellaria barbata D. Don on human breast carcinoma bone metastasis via downregulating PTHrP pathway. 2018, 41(6):3137-3146.

14. Zhao B, Hui X, Jiao L, Bi L, Wang L, Huang P, Yang W, Yin Y, Jin S, Wang C et al: A TCM Formula YYWY Inhibits Tumor Growth in Non-Small Cell Lung Cancer and Enhances Immune-Response Through Facilitating the Maturation of Dendritic Cells. 2020, 11:798.

15. Nagl N, Patsialou A, Haines D, Dallas P, Beck G, Moran E: The p270 (ARID1AVSMARCF1) subunit of mammalian SWI/SNF-related complexes is essential for normal cell cycle arrest. Cancer research 2005, 65(20):9236-9244.

16. Kastan MB, Bartek J: Cell-cycle checkpoints and cancer. Nature 2004, 432(7015):316-323.

17. Beauparlant $\mathrm{P}$, Shore G: Therapeutic activation of caspases in cancer: a question of selectivity. Current opinion in drug discovery \& development 2003, 6(2):179-187.

18. Valastyan S, Weinberg RA: Tumor metastasis: molecular insights and evolving paradigms. Cel/ 2011, 147(2):275-292.

19. Takamatsu SJJonm: Naturally occurring cell adhesion inhibitors. 2018, 72(4):817-835.

20. Rebello R, Oing C, Knudsen K, Loeb S, Johnson D, Reiter R, Gillessen S, Van der Kwast T, Bristow R: Prostate cancer. Nature reviews Disease primers 2021, 7(1):9.

21. Han W, Shi Y, Su J, Zhao Z, Wang X, Li J, Liu H: Virtual Screening and Bioactivity Evaluation of Novel Androgen Receptor Antagonists From Anti-PCa Traditional Chinese Medicine Prescriptions. Frontiers in chemistry 2020, 8:582861.

22. Wang N, Xu L, Wang J, Yu X, Chu L, Deng S, Ge F, Li H: Traditional Chinese medicine on treating pain caused by prostate cancer: A systematic review and meta-analysis. Medicine 2019, 98(44):e17624.

23. Yang A, Zhao Y, Wang Y, Zha X, Zhao Y, Tu P, Hu Z: Huaier suppresses proliferative and metastatic potential of prostate cancer PC3 cells via downregulation of Lamin B1 and induction of autophagy. Oncology reports 2018, 39(6):3055-3063.

24. Basu A: The interplay between apoptosis and cellular senescence: Bcl-2 family proteins as targets for cancer therapy. Pharmacology \& therapeutics 2021:107943.

25. Spitz A, Zacharioudakis E, Reyna D, Garner T, Gavathiotis E: Eltrombopag directly inhibits BAX and prevents cell death. Nature communications 2021, 12(1):1134.

26. Shen X, Burguillos M, Osman A, Frijhoff J, Carrillo-Jiménez A, Kanatani S, Augsten M, Saidi D, Rodhe $\mathrm{J}$, Kavanagh E et al: Glioma-induced inhibition of caspase-3 in microglia promotes a tumorsupportive phenotype. Nature immunology 2016, 17(11):1282-1290.

27. Grinkevich V, Nikulenkov F, Shi Y, Enge M, Bao W, Maljukova A, Gluch A, Kel A, Sangfelt O, Selivanova $\mathrm{G}$ : Ablation of key oncogenic pathways by RITA-reactivated p53 is required for efficient apoptosis. 
Cancer cell 2009, 15(5):441-453.

28. Quintanal-Villalonga A, Taniguchi H, Zhan Y, Hasan M, Chavan S, Meng F, Uddin F, Allaj V, Manoj P, Shah $\mathrm{N}$ et al: Comprehensive molecular characterization of lung tumors implicates AKT and MYC signaling in adenocarcinoma to squamous cell transdifferentiation. 2021, 14(1):170.

29. Tehranian C, Fankhauser L, Harter P, Ratcliffe C, Zeiner P, Messmer J, Hoffmann D, Frey K, Westphal $\mathrm{D}$, Ronellenfitsch $\mathrm{M}$ et al: The PI3K/Akt/mTOR pathway as a preventive target in melanoma brain metastasis. 2021.

30. Lambert A, Weinberg R: Linking EMT programmes to normal and neoplastic epithelial stem cells. Nature reviews Cancer 2021, 21(5):325-338.

31. Yang J, Antin P, Berx G, Blanpain C, Brabletz T, Bronner M, Campbell K, Cano A, Casanova J, Christofori $\mathrm{G}$ et al: Guidelines and definitions for research on epithelial-mesenchymal transition. Nature reviews Molecular cell biology 2020, 21(6):341-352.

32. De Craene $B$, Berx G: Regulatory networks defining EMT during cancer initiation and progression. Nature reviews Cancer 2013, 13(2):97-110.

33. Medová M, Medo M, Hovhannisyan L, Muñoz-Maldonado C, Aebersold D, Zimmer Y: DNA-PK in human malignant disorders: Mechanisms and implications for pharmacological interventions. Pharmacology \& therapeutics 2020, 215:107617.

34. Wang X, Xu H, Cheng C, Ji Z, Zhao H, Sheng Y, Li X, Wang J, Shu Y, He Y et al: Identification of a Zeb1 expressing basal stem cell subpopulation in the prostate. Nature communications 2020, 11(1):706.

35. Wang H, Wu Q, Liu Z, Luo X, Fan Y, Liu Y, Zhang Y, Hua S, Fu Q, Zhao M et al: Downregulation of FAP suppresses cell proliferation and metastasis through PTEN/PI3K/AKT and Ras-ERK signaling in oral squamous cell carcinoma. Cell death \& disease 2014, 5:e1155.

36. Zhou Z, Wang S, Song C, Hu Z: Paeoniflorin prevents hypoxia-induced epithelial-mesenchymal transition in human breast cancer cells. OncoTargets and therapy 2016, 9:2511-2518.

37. Jing Cao, Zhang G, Liu Z, Xu Q, Li C, Cheng G, Shi R: Peroxidasin promotes diabetic vascular endothelial dysfunction induced by advanced glycation end products via NOX2/HOCl/Akt/eNOS pathway. Redox biology 2021, 45:102031.

38. Sarkar C, Goswami S, Basu S, Chakroborty DJC: Angiogenesis Inhibition in Prostate Cancer: An Update. 2020, 12(9).

\section{Figures}

Figure 1

Total ion chromatograph of SBD in positive ion modes. 
High-performance liquid chromatography (HPLC) analysis of SBD and control sample. There were four

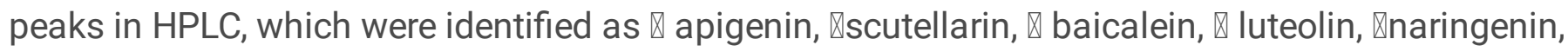
『wogonin.

\section{Figure 2}

\section{SBD inhibits the proliferation of PCa cells}

Cell viabilities were measured by CCK-8 assay after treatment with indicated concentrations of SBD for $24 \mathrm{~h} \mathrm{(a)} \mathrm{and} 48 \mathrm{~h}$ (b). The cell proliferation and apoptosis of PC-3 (c) and DU145 (d) cell lines with or without SBD treatment determine by EdU and Hoechst staining. Cell cycle of PC-3 (e) and DU145 (f) cell lines measured by flow cytometry analysis after SBD treatment for $48 \mathrm{~h}$. The colony formation of PC-3 (g) and DU145 $(h)$ cell lines with or without SBD treatment $(n=4), p<0.05\left(^{*}\right)$.

\section{Figure 3}

\section{SBD inhibited PCa cell apoptosis through Akt/PI3K signaling pathway}

Cell apoptosis measured by flow cytometry after SBD treatment with different concentrations for $48 \mathrm{~h}$ in PC-3 (a) and DU145 (b) cell lines; Protein expression levels of Cleaved caspase-3, Bcl-2 and Bax in PC-3 and DU145 cells determined by western blotting assay(c); Relative protein expression levels of p-Akt, Akt, $\mathrm{p}-\mathrm{PI} 3 \mathrm{~K}$ and PI3K with or without SBD treatment determined by western blotting assay $(n=4), p<0.05(*)$. 
a
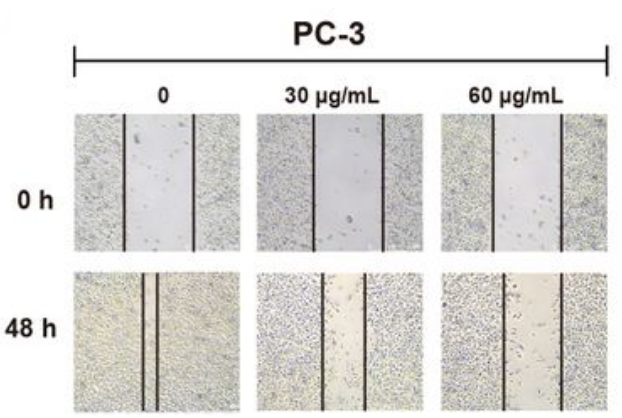

C

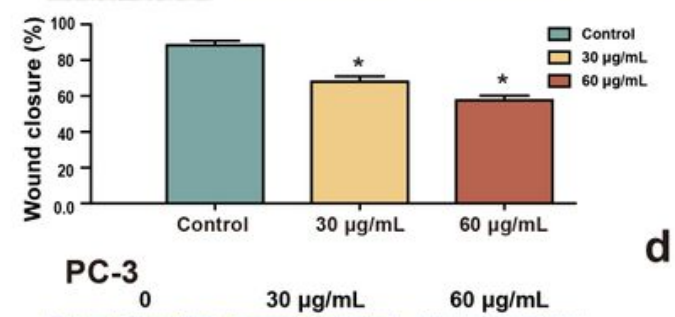

b
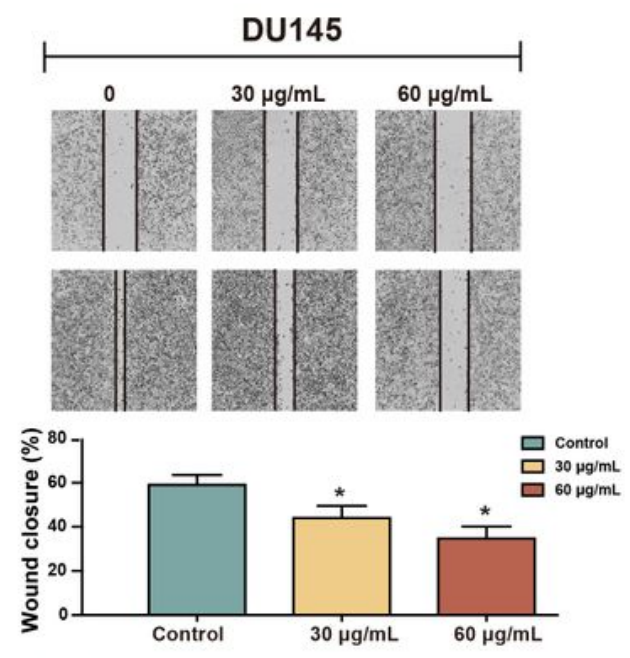

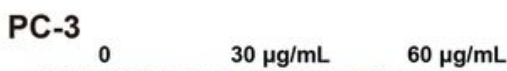

Figure 4

Effects of SBD on the migration and invasion capabilities in PC3 and DU145 cells.

Wound-healing assay was conducted to examine cellular migration of PC-3 (a) and DU145 (b) at 0 and $48 \mathrm{~h}$; Cell migration (c) and invasion (d) of PC3 and DU145 cells that underwent SBD treatment was 
determined by transwell assay; (e) The expression of EMT-related proteins in PC3 and DU145 cells with or without SBD treatment was determined by western blotting assay $(n=4), p<0.05\left(^{*}\right)$.

\section{Figure 5}

\section{SBD inhibited PCa cell vitality and invasion target Akt}

Cell viabilities of PC-1 (a) and DU145 (b) cell lines pre-treated by MK2206 were measured by CCK-8 assay after treatment with indicated concentrations of SBD. Cell invasion of PC-1 (c) and DU145 (d) cell lines that underwent SBD treatment was determined by transwell assay $(n=4), p<0.05(*)$.

\section{Figure 6}

\section{SBD inhibited PCa cell EMT-related protein target Akt}

The relative protein expression levels of p-Akt, Akt, and EMT-related proteins in PC-3 (a) and DU145 (b) cell lines with or without SBD or MK2206 treatment was determined by western blotting assay $(n=5), p<$ $0.05\left({ }^{*}\right)$.

\section{Figure 7}

\section{SBD inhibited tumour growth in xenograft mice model}

(a) Xenograft tumors imaging of 22RV1 in different groups at the end of experiments; (b) Administration of different concentration SBD decreased the tumour weight (g); (c) Administration of different concentration SBD decreased the tumour volume $\left(\mathrm{mm}^{3}\right)$; (d) Administration of different concentration SBD attenuated the tumour tissue damage measured by H\&E staining; (e) The expression of Ki67 measured by IHC staining in tumour tissues from xenograft C57BL/ 6 mice $(n=5), p<0.05$. 
Figure 8

\section{SBD inhibited EMT through Akt/PI3K signalling pathway in xenograft mice model}

(a) The relative protein expression levels of p-Akt, Akt, p-PI3K and PI3K in xenograft tumours determined by western blotting assay; (b) The EMT-related proteins in xenograft tumours determined by IHC staining $(n=3), p<0.05(*)$.

\section{Figure 9}

Effects of conditional media (CM) from control- or SBD-treated PCa cells on tube formation, viability of HUVEC

(a) PCa cells were treated without or with SBD at the indicated doses for $48 \mathrm{~h}$. The conditioned media (CM) was then doses for $24 \mathrm{~h}$. The conditional media (CM) was then harvested and applied to HUVEC cultured in Matrigel-coated plates for an additional $4 \mathrm{~h}$. Changes in cell morphology were captured using an microscope. (b) HUVECs were treated without or with SBD for $4 \mathrm{~h}$, followed by measurement of cell viability by CCK8 (c) PCa cells were treated without or with PMA (10 nM), in the absence or presence of SBD for $4 \mathrm{~h}$. The CM was then collected and applied to HUVEC changes in HUVEC tube formation were captured using an inverted microscope. $(n=5), p<0.05\left(^{*}\right)$. 